\title{
International Journal of

\section{A New Idea for Stroke Rehabilitation: Bilateral Transfer Analysis from Healthy Hand to the Paretic One with a Randomized and Controlled Trial}

Carlo Domenico Ausenda*, Giovanni Togni, Marco Biffi, Simona Morlacchi, Mariagrazia Corrias and Giovanna Cristoforetti

General Hospital San Carlo Borromeo, Milan, Italy

*Corresponding author: Carlo Domenico Ausenda, General Hospital San Carlo Borromeo, Milan, Italy, Tel: 0240222661; Fax: 02-4022-2327; E-mail: ausenda.carlo@sancarlo.mi.it

Rec date: 03 Dec 2013; Acc date: 24 Feb 2014; Pub date: 27 Feb 2014

Copyright: (c) 2014 Ausenda CD, et al. This is an open-access article distributed under the terms of the Creative Commons Attribution License, which permits unrestricted use, distribution, and reproduction in any medium, provided the original author and source are credited.

\begin{abstract}
Background: Bilateral transfer of a motor skill is a phenomenon according to which, one hand can "teach" a skill to the other hand. In this research, controlled and randomized, we tested the ability of bilateral transfer (BT) to improve the coordination of the paretic hand in patients that suffered a stroke, with the aim of restricting the field for further research on the impact of BT in rehabilitation, we analyzed the differences in the expression of BT phenomenon among sexes and side of hemyparesis.
\end{abstract}

Methods: 34 right-handed patients, at the end of the rehabilitation period, were randomly divided into two groups: test and control. They all had a stroke in a single hemisphere in the previous six months and they were selected by a physical examination, the time elapsed from the stroke and cognitive requirements. The experiment consisted in training the healthy hand of each patient from the test group to execute the nine hole peg test (NHPT) 10 times a day, for 3 consecutive days, and then test the paretic hand with the same test and with bimanual tasks. The control group was not trained but went through the same analysis.

Results: In the test group we found that the execution speed of NHPT with the paretic hand, after training the healthy hand, was on average $22.6 \%$ faster than the value recorded at baseline. Meanwhile, no significant difference was found in the control group. The analysis showed a greater impact of BT among male patients, who were in average $31 \%$ faster than controls, and in non dominant paretic hands, that were $30 \%$ faster after training.

Conclusions: BT was present in hemiparethic patients with moderate stroke after a short time span, it was more evident among male subjects, and from the dominant hand to the paretic non-dominant one.

Keywords: Hemiparesis; Stroke; Upper extremity paresis; Motor control laterality; Randomized; Controlled trial

\section{Introduction}

Stroke is a leading cause of adult disability and, although stroke can cause deficits in a number of neurological domains, the most commonly affected is the motor system [1]. Given the central role that hand movements normally play in human existence [2], much attention in rehabilitation research has been focusing on restoring hand motor function after a stroke [3]. A recurring theme is that interventions emphasizing intense, active repetitive movements are of high value in this regard [1,4]. These increase strength, accuracy and functional use when applied to subjects with paresis due to stroke $[1,5]$. Moreover they showed the beneficial effects of motivation, by upper limb constraint [4], and the importance of visual control [5].

In the early twentieth century the phenomenon describing how the performance of a skill with one hand seemed to "teach" the same skill to the other hand was observed. E. J. Swift, in 1903 [6] studied the transfer of juggling skill from one hand to the other (hand-to-hand), without previous training. Hand-to-hand transfer was defined "bilateral" transfer (BT). Transfer not only occurred from hand-tohand, but also from hand-to-foot and from foot-to-foot and from feetto-hands [7]. Recently BT of a sensitive experience was shown to be present for fingertip forces anticipation, in children with hemiplegic cerebral palsy [8]. Since BT occurred only when attended by attention, motivation and the performance of repetitive movements [6,7], i.e. all conditions required for the rehabilitation of stroke patients, we speculated that training of the healthy hand with a highly repetitive task might "facilitate" the training of the paretic hand, and that this might also have an influence on bi-manual skills. We studied the existence of the phenomenon of BT in a pilot study on a patient [9], and we obtained encouraging results after short term and after 4 months follow up, on his paretic hand dexterity.

As the first published, controlled and randomized research deals with [10], we demonstrated that BT was present, in a short-term, in hemiparethic patients with stroke, improving their paretic hand dexterity.

A recent study on stroke rehabilitation demonstrated that the arm use after right or left hemiparesis is influenced by manual preference [11], but studies about BT [12] revealed that BT is common in healthy people and it is not related to the dominant hand, although it is more frequent from the dominant hand to the other one. Moreover the analysis on sex differences in BT proved it is more common among men [12].

In order to proceed to further research on the utilization of BT in stroke rehabilitation we needed to understand if, among right-handed stroke patients, the BT phenomenon is more evident in right or left 
Citation: Ausenda CD, Togni G, Biffi M, Morlacchi S, Corrias M, et al. (2014) A New Idea for Stroke Rehabilitation: Bilateral Transfer Analysis from Healthy Hand to the Paretic One with a Randomized and Controlled Trial. Int J Phys Med Rehabil S3: 008. doi: 10.4172/2329-9096.S3-008

Page 2 of 8

hands hemiparesis and verify the differences among sexes. The previously applied protocol [10] was enlarged to a greater number of patients with the aim of reaching a result that could clarify those questions, with a statistical significance.

\section{Materials and Methods}

\section{Participants}

34 patients that suffered a recent stroke, aged $66,1 \pm 11,7$ years, with upper extremity paresis were recruited for this randomized controlled trial: 16 were male, all were right-hand-dominant before stroke; 32 were ischaemic strokes, 2 were cerebral hemorragic intraparenchimal strokes; 22 were in the left hemisphere and 12 in the contralateral. The project was approved by the review committee of the institution and the subjects gave informed consent. The patients were randomly assigned to the test group or to the control group, 17 in each one, following simple randomization procedures (computerized random numbers). In the attempt of examining homogeneous groups patients had to fulfill physical examination and cognitive requirements (Tables 1 and 2), since the physical examination inclusion criteria were very selective, the clinical appearance of the patients was mild or moderate hemiparesis. The sample size was decided planning the experimental design, in order to achieve a statistical significance, matching previous results with the Consort Statement instructions for non pharmaceutical RCT [10].

The inclusion and exclusion criteria are summarized in Tables 1 and 2. The patients were recruited in the project when their rehabilitation ended, in order to avoid the influence of differences in the exercises previously performed, and they all had suffered a stroke within the last 6 months. The procedures described below were applied to each patients by the same examiner, who was not blinded.

\footnotetext{
Written informed consent

Active wrist extension of the affected upper limb of at least $10^{\circ}$

Active metacarpo-phalangeal extension of at least $10^{\circ}$

Active inter-phalangeal extension of at least $10^{\circ}$

Active abduction and extension of the thumb and of two more fingers of at least $10^{\circ}$

Active movements are to be repeated at least three times in a minute

Passive abduction and flexion of the shoulder of at least $90^{\circ}$

Passive extrarotation of the shoulder at least $45^{\circ}$

Maximum limitation of the passive extension of the elbow $30^{\circ}$

Passive supination of the forearm from neutral position at least $45^{\circ}$

Passive pronation of the of the forearm from the neutral position at least $45^{\circ}$

Passive extension of the wrist and fingers do not cause a flexion of the metacarpophalangeal joints of more then $30^{\circ}$
}

Table 1: Inclusion criteria

\section{Procedures}

The day of recruitment, in order to verify the homogeneity of the patients and the inclusion/exclusion criteria, all were submitted to: Mini Mental State Examination [13]; Barthel Index [14]; physical examination of the upper limb, with recordings of the Ashworth Scale of muscular tone [15] and of the articular range of passive and active motion (Table 1); a hand preference test [16].
The main exercise for both groups was the Nine Hole Peg Test (NHPT) [17] (Figure 1), adapted to our purpose with a larger grid, and smaller pegs, done as fast as possible with the paretic and the healthy hand at the beginning and at the end of the project (primary outcome measure), recorded in seconds. We chose the NHPT [18] (Figure 1) for evaluation of the single manual function, because this movement involves the coordination of the whole upper limb: opposition of the thumb, flexion-extension of the fingers, prono-supination of the wrist, flexion-extension of the elbow, requiring stability of the shoulder, and also because it gives a very precise measure in seconds of the single hand dexterity [17]. For the same reasons we chose NHPT for the experimental training, as a highly repetitive exercise.

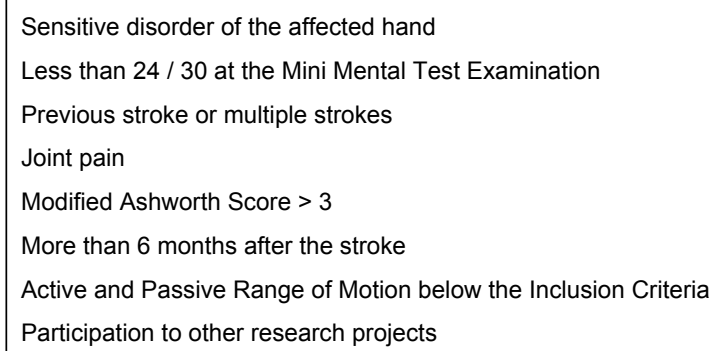

Table 2: Exclusion criteria

The patients in the test group, had to repeat consecutively 10 times each day, for three consecutive days the exercise of doing the nine hole peg test with the normal hand, carefully and paying attention to the various parts of the movement.

The patients in the control group did not train the normal hand, and were re-tested on the third day.

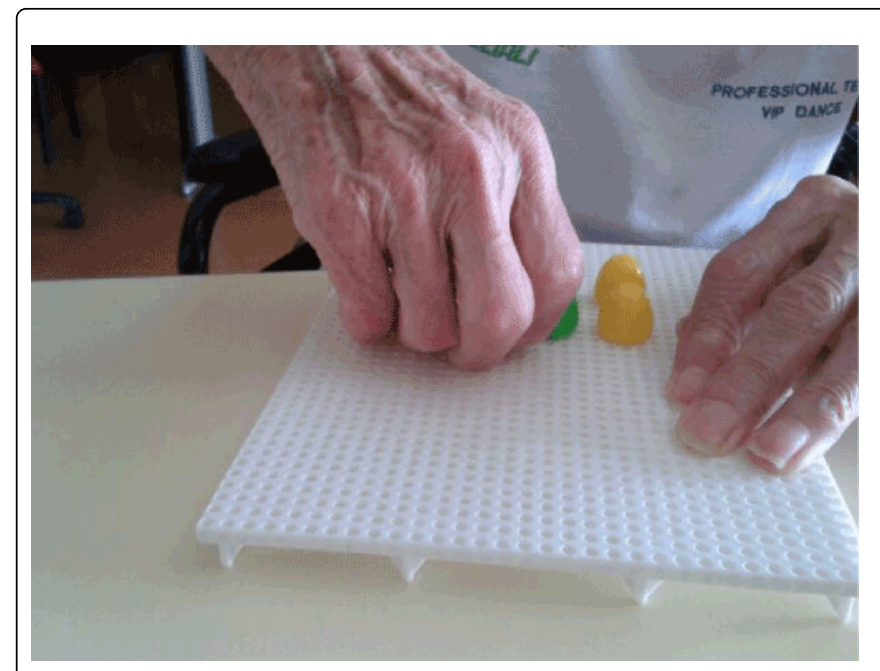

Figure 1: NHPT. Nine pegs must the inserted in to nine hole of a grid and then removed.

The patients in both groups had their bimanual hand coordination re-tested by the items 10 (Figure 2), 11 (Figure 3), and 14 (Figure 4) of The Sollermann's Test [18] at the end of the project (secondary outcome measure). 
Citation: Ausenda CD, Togni G, Biffi M, Morlacchi S, Corrias M, et al. (2014) A New Idea for Stroke Rehabilitation: Bilateral Transfer Analysis from Healthy Hand to the Paretic One with a Randomized and Controlled Trial. Int J Phys Med Rehabil S3: 008. doi: 10.4172/2329-9096.S3-008

Page 3 of 8

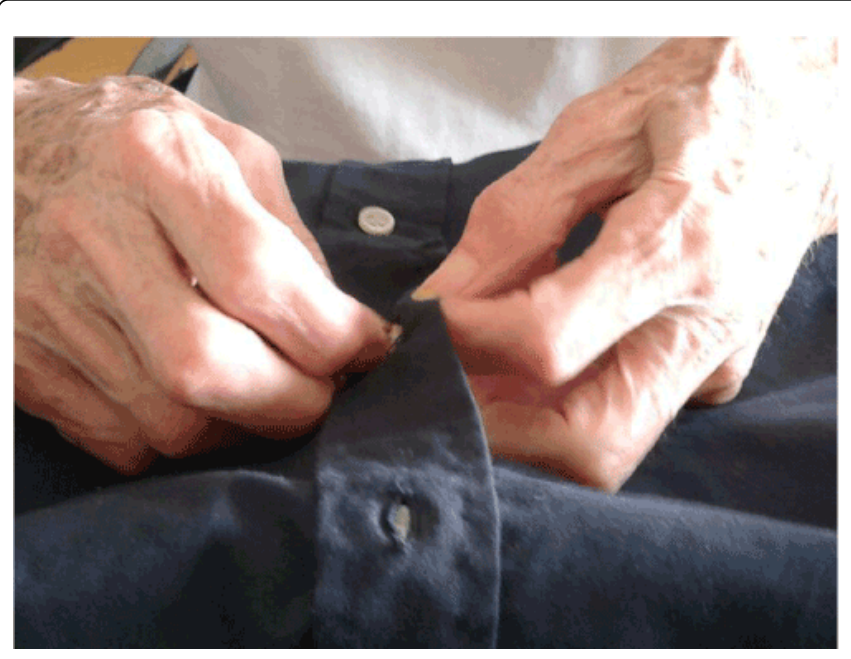

Figure 2: Sollerman test, item 10. The patient has to button with both hands 4 buttons of a shirt, and then undo them

The Sollerman's Test is a standardised hand function test based on seven of the eight most common hand grips. The test consists of 20

\begin{tabular}{|c|c|c|c|c|c|c|c|}
\hline Patient & Site of brain lesion & Sex & age & mmse & barthel & Ashworth & Days after stroke \\
\hline PT & Isc R basal ganglia & $\mathrm{F}$ & 83 & 26 & 55 & 2 & 33 \\
\hline RL & Isc L parietal lobe & $\mathrm{F}$ & 83 & 30 & 95 & 1 & 48 \\
\hline GG & Isc $L$ frontal lobe & $\mathrm{F}$ & 68 & 24 & 100 & 2 & 68 \\
\hline CR & Isc L basal ganglia & $\mathrm{F}$ & 83 & 27 & 100 & 1 & 35 \\
\hline TR & Isc R temporal lobe & M & 73 & 24 & 60 & 2 & 175 \\
\hline SD & Isc $R$ frontal lobe & M & 52 & 30 & 100 & 2 & 21 \\
\hline $\mathrm{DE}$ & Isc R parietal lobe & M & 70 & 28 & 100 & 1 & 142 \\
\hline DA & Isc $L$ frontal lobe & M & 53 & 25 & 100 & 2 & 145 \\
\hline CG & Isc $L$ temporal lobe & M & 60 & 28 & 100 & 1 & 32 \\
\hline GG & Isc $L$ frontal lobe & M & 53 & 30 & 95 & 2 & 58 \\
\hline$B C$ & Isc L basal ganglia & $\mathrm{F}$ & 64 & 28 & 65 & 0 & 74 \\
\hline TF & Hem L frontal lobe & M & 66 & 27 & 98 & 2 & 12 \\
\hline DC & Isc L parietal lobe & $\mathrm{F}$ & 78 & 28 & 90 & 0 & 138 \\
\hline TL & Isc $L$ frontal lobe & M & 80 & 35 & 85 & 0 & 54 \\
\hline VG & Isc $L$ frontal lobe & $\mathrm{s}$ & 69 & 26 & 100 & 0 & 46 \\
\hline OV & Isc $L$ fronto-temporal & M & 66 & 27 & 90 & 1 & 15 \\
\hline GR & Isc $L$ frontal lobe & $\mathrm{F}$ & 60 & 28 & 97 & 1 & 55 \\
\hline
\end{tabular}

Table 3: Description of the Test group

Site of brain lesion: lobe mainly involved; Isc: Ischaemia, Hem: Hemorrage R: right, L: left; Age: years. Sex. F: female, M: male ; Mmse: Mini Mental State Examination, points (0-30); Barthel: Barthel Index, activities of daily living, that can be performed with one or two hands. The test gives 4 points to assess the quality of the movement ( 1 poor, 4 good). The patients in both groups (described in Tables 3 and 4) had their bimanual hand coordination tested by the items 10 (buttoning and unbuttoning a shirt with both hands) (Figure 2), item 11 (the patient has to cut in 4 parts a piece of fresh plastiline) (Figure 3) and item 14 (the patient has to fold a sheet of paper in 4 and put it tightly into an envelope) (Figure 4) of the Sollermann's Test (18) adapted for our purpose by the recording of the time of execution in seconds, before undergoing the exercise, and not giving points to the quality of the movement, in order to amplify the relevance of eventual variations. The patient flow in the experimental design is described in Figure 5.

The procedures were administered in the examiner's office in a general hospital rehabilitation facility for inpatients and outpatients.

\section{Statistical analysis}

The demographic variables were analysed with descriptive statistics calculating the mean and the standard deviation for parametrical data, meanwhile for the non parametrical data, frequencies were calculated.

The homogeneity of the two groups was evaluated using unpaired Student $T$ test for age, MMSE, Barthel Index and Ashworth scale. 
Citation: Ausenda CD, Togni G, Biffi M, Morlacchi S, Corrias M, et al. (2014) A New Idea for Stroke Rehabilitation: Bilateral Transfer Analysis from Healthy Hand to the Paretic One with a Randomized and Controlled Trial. Int J Phys Med Rehabil S3: 008. doi: 10.4172/2329-9096.S3-008

Page 4 of 8

\begin{tabular}{|c|c|c|c|c|c|c|c|}
\hline Patient & Site of brain lesion & Sex & Age & Mmse & Barthel & Ashworth & Days after stroke \\
\hline VR & Isc L parietal lobe & M & 59 & 28 & 95 & 0 & 30 \\
\hline MS & Isc $L$ temporal lobe & M & 68 & 28 & 100 & 1 & 63 \\
\hline$A G$ & Isc $R$ parietal lobe & $\mathrm{F}$ & 72 & 28 & 55 & 1 & 33 \\
\hline DP & Isc $L$ frontal lobe & M & 77 & 27 & 90 & 1 & 19 \\
\hline SR & Isc L parietal lobe & $\mathrm{F}$ & 61 & 25 & 60 & 1 & 65 \\
\hline LS & Isc R temporal lobe & M & 51 & 30 & 95 & 1 & 170 \\
\hline NE & Isc $R$ parietal lobe & $M$ & 84 & 27 & 65 & 2 & 132 \\
\hline SP & Isc L basal ganglia & $\mathrm{F}$ & 46 & 30 & 100 & 1 & 92 \\
\hline MA & Isc $R$ parietal lobe & $\mathrm{F}$ & 46 & 30 & 100 & 2 & 171 \\
\hline OD & Isc $L$ temporal lobe & $\mathrm{F}$ & 49 & 30 & 95 & 1 & 103 \\
\hline MG & Isc $L$ frontal lobe & $\mathrm{F}$ & 58 & 30 & 99 & 1 & 24 \\
\hline CE & Isc L parietal lobe & $\mathrm{F}$ & 80 & 24 & 65 & 1 & 40 \\
\hline SL & Isc $\mathrm{R}$ basal ganglia & $\mathrm{F}$ & 69 & 30 & 92 & 2 & 29 \\
\hline FA & Isc $L$ frontal lobe & $\mathrm{F}$ & 81 & 25 & 65 & 1 & 23 \\
\hline RA & Isc. $R$ frontal lobe & M & 60 & 30 & 93 & 1 & 102 \\
\hline MM & Hem $L$ frontal lobe & $\mathrm{F}$ & 72 & 30 & 94 & 2 & 85 \\
\hline $\mathrm{CN}$ & Isc $L$ frontal lobe & $\mathrm{F}$ & 52 & 30 & 98 & 1 & 71 \\
\hline
\end{tabular}

Table 4: Description of the control group

Table legend: Site of brain lesion: lobe mainly involved; Isc: Ischaemia; Hem Hemorrage; R: right, L: left; Age: years. Sex: F: female, M: male; Mmse: Mini Mental State Examination, points (0-30); Barthel: Barthel Index, percentage (0-100\%); Ashworth: Modified Ashworth Scale, points (0-5); Days after stroke: days elapsed from the pathologic event to the beginning of the rehabilitation.

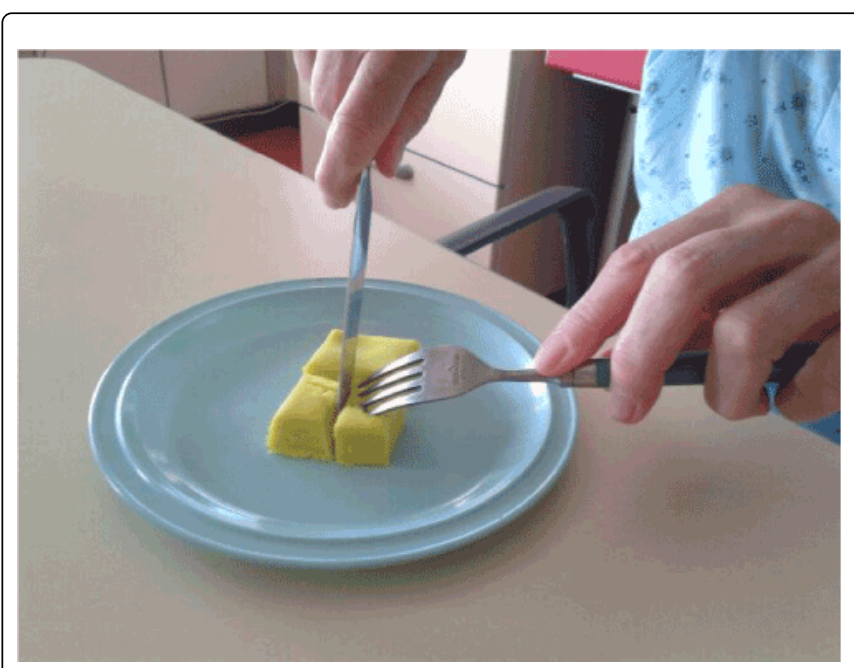

Figure 3: Sollerman test, item 11. The patient has to cut in 4 parts a piece of fresh plastiline

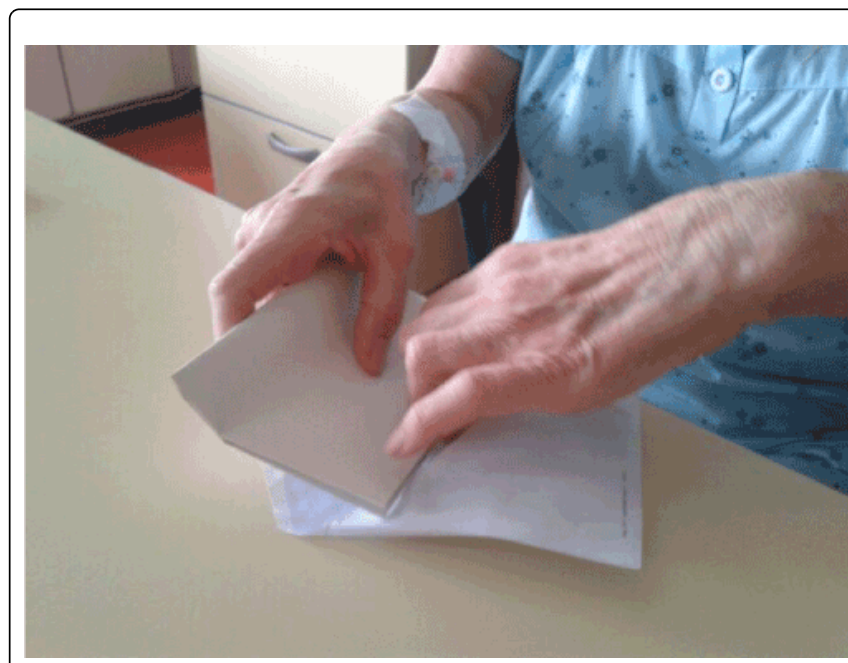

Figure 4: Sollerman test, item 14. The patient has to fold a sheet of paper in 4 and put it tightly into an envelope

The whole group is composed by 34 patients (16 men and 18 women); 7 women and 10 men are in the exercise group.

We estimated an average of times of execution of manual and bimanual tests with related measures of changeability, dividing into sex, group (exercise/non-exercise) and hand (paretic/non-paretic and left/ right). 
Citation: Ausenda CD, Togni G, Biffi M, Morlacchi S, Corrias M, et al. (2014) A New Idea for Stroke Rehabilitation: Bilateral Transfer Analysis from Healthy Hand to the Paretic One with a Randomized and Controlled Trial. Int J Phys Med Rehabil S3: 008. doi: 10.4172/2329-9096.S3-008

Page 5 of 8

Appropriate non parametric test (Wilcoxon/Mann-Whitney T test for dependent sample with unilateral choice) can be used to estimate the hypothesis concerning the reduction of times of movement execution in a second, and to create a sequence.

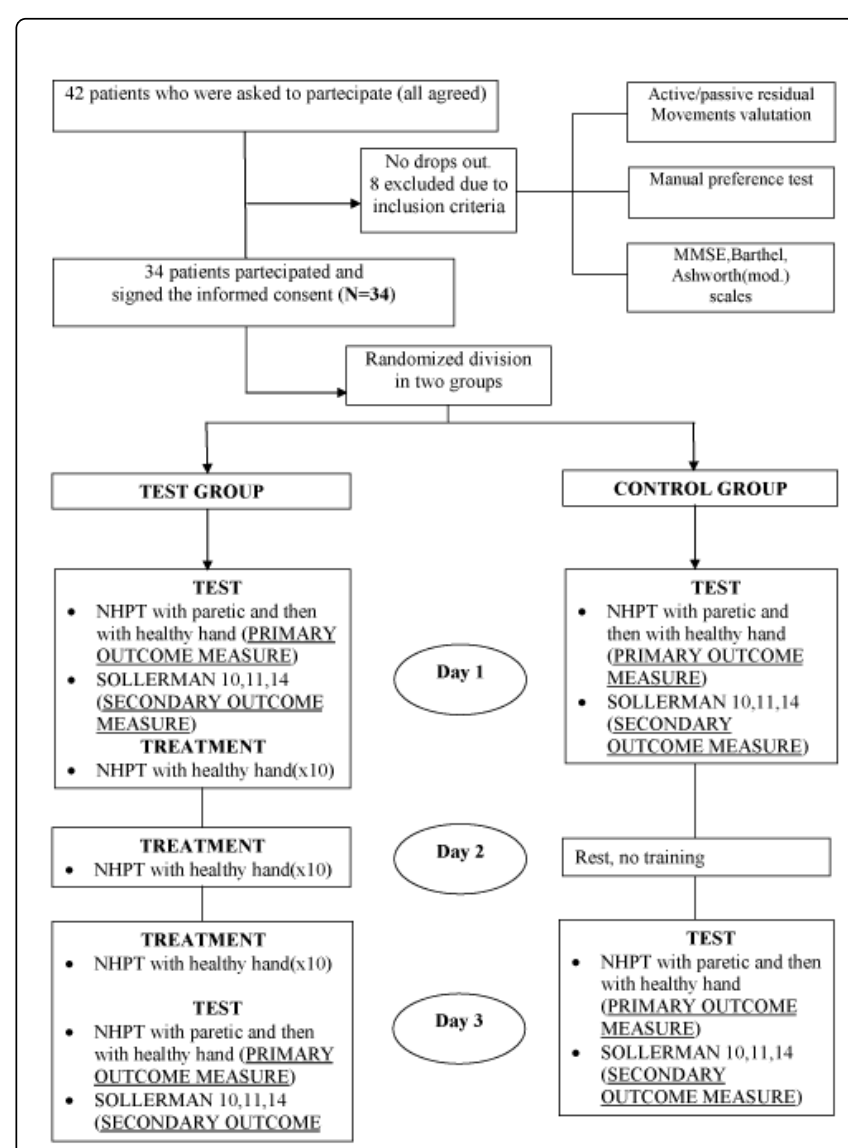

Figure 5: patient's flow in the trial design

\section{Results}

In Table 5 are summarized the results of the execution times for NHPT and the related standard deviation, divided into sex, group belonging and execution with paretic or healthy hand.

Results of execution times for NHPT and the related standard deviation, divided into sex, group belonging and execution with paretic or healthy hand.

There was a significative difference between the performance in the first and the second survey for each group in healthy and paretic hand.

We found a statistically significant variation among sexes in exercise group.

In the men group from the first survey to the second one we found a reduction of about 5 seconds in the healthy hand performance $(-13 \%, p$-value 0.078$)$ and of about 26 seconds in the paretic hand $(-31 \%, \mathrm{p}$-value 0.0039$)$; in the women group the reduction was 8 second in the healthy hand $(-10 \%$, p-value 0.0156$)$ and 20 seconds in paretic (-20\%, p-value 0.0156$)$.

There is a minor improvement in no-exercise group (Graph 1).

\begin{tabular}{|c|c|c|c|c|}
\hline & & \multicolumn{3}{|c|}{$\begin{array}{l}\text { NHPT Average time in seconds (+SD) } \\
\text { Before/After }\end{array}$} \\
\hline & & Test Group & Control Goup & Total \\
\hline \multirow[t]{4}{*}{ Men } & Healthy & $42.0(9.65)$ & 43.6 (13.24) & $42.6(10.7)$ \\
\hline & & $36.9(11.3)$ & $43.2(13.5)$ & $39.3(12.1)$ \\
\hline & Paretic & $109.4(54.6)$ & $126.2(61.3)$ & $115.8(55.4)$ \\
\hline & & $82.5(48.5)$ & $127.4(62.7)$ & $99.8(56.6)$ \\
\hline \multirow[t]{4}{*}{ Women } & Healthy & 49.8 (5.9) & $47.1(23.9)$ & $48.4(17.3)$ \\
\hline & & $41.2(7.7)$ & $45.4(25.7)$ & $43.5(19.0)$ \\
\hline & Paretic & $102.1(55.8)$ & $77.7(48.0)$ & $89(51.1)$ \\
\hline & & $83.7(59.1)$ & $71.3(45.9)$ & $77.0(50.5)$ \\
\hline \multirow{4}{*}{$\begin{array}{l}\text { Total } \quad \text { (Men } \\
\text { +Women) }\end{array}$} & Healthy & $45.4(8.9)$ & $45.7(19.4)$ & $45.5(14.4)$ \\
\hline & & 38.7 (9.8) & $44.5(20.7)$ & $41.4(15.7)$ \\
\hline & Paretic & $106.3(53.1)$ & $97.9(57.0)$ & $102.4(54.0)$ \\
\hline & & $83.0(51.1)$ & $94.7(58.5)$ & $88.4(55.8)$ \\
\hline
\end{tabular}

Table 5: results of execution times for NHPT
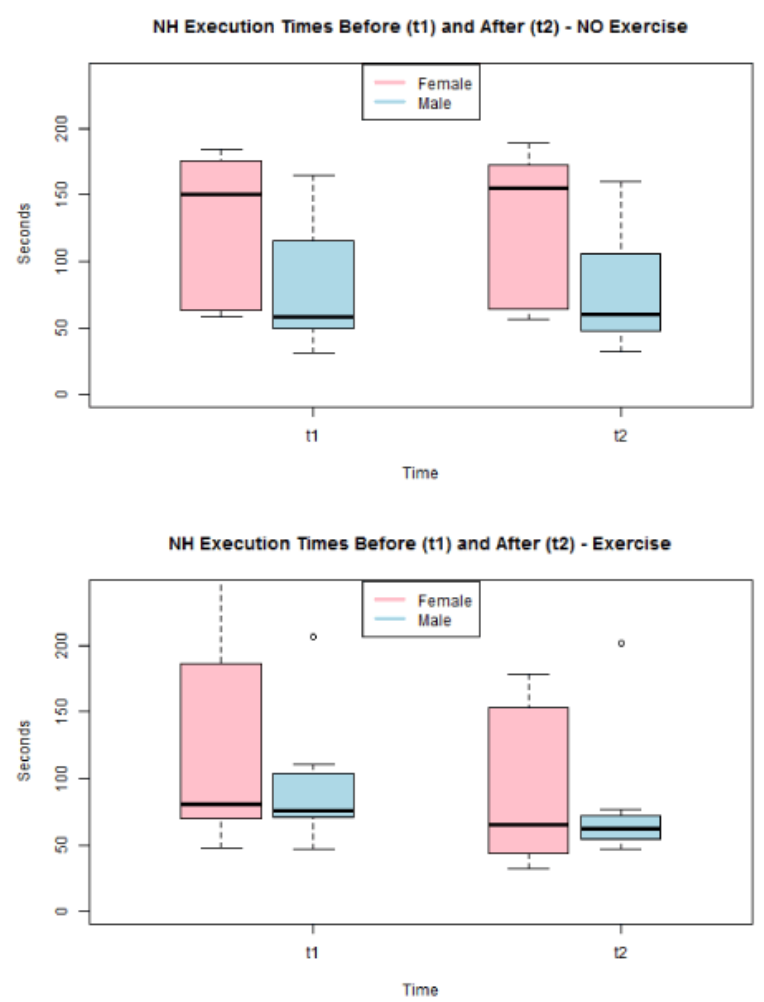

Graph 1: boxplot concerning time results in NHPT execution sex related

Regarding to the paretic hand we observed a reduction in the average performances in both groups with left hemiparesis: left hemiparesis patients (12 patients) improved with and without exercise (with exercise: $\sim 30$ seconds, $-30 \%$ p-value 0.048 ; without exercise: $\sim 24$ seconds, $-18 \%$, p-value 0.03 ). 
Citation: Ausenda CD, Togni G, Biffi M, Morlacchi S, Corrias M, et al. (2014) A New Idea for Stroke Rehabilitation: Bilateral Transfer Analysis from Healthy Hand to the Paretic One with a Randomized and Controlled Trial. Int J Phys Med Rehabil S3: 008. doi: 10.4172/2329-9096.S3-008

Page 6 of 8

Right hemiparesis patients didn't seem to improve inthe Noexercise group, but there was a reduction of $\sim 20$ seconds in the exercise group patients (-27\%, p-value 0.007).

Graph 2 summarizes data concerning the exercise group.

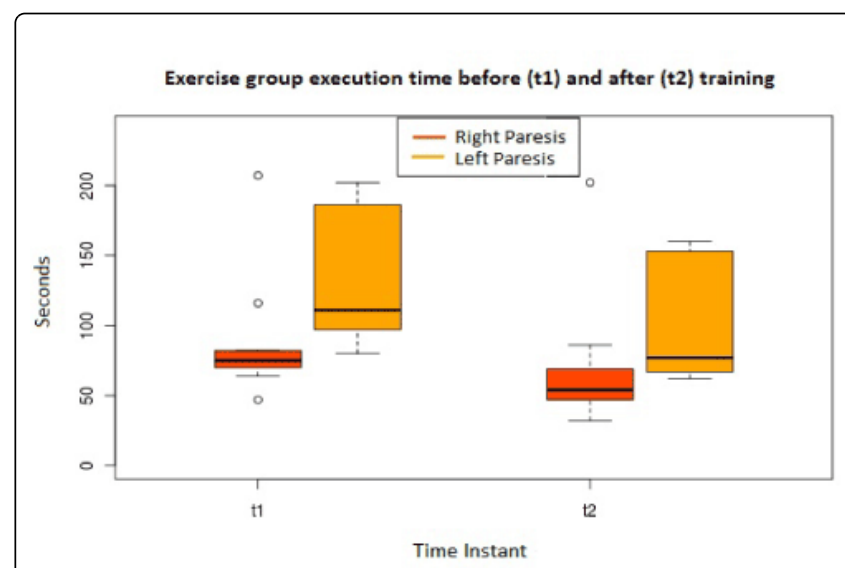

Graph 2: contains boxplot concerning time results in NHPT in right and left hemipharesis

The bimanual tests (Sollerman's Item 10, 11, 14) measured in seconds, showed an improvement in the performances in item 10 and 11 of the women exercise group (item 10: 13 seconds, -13\% p-value 0.047; item 11: $\sim 4$ seconds, $-11 \%$ p-value 0.037$)$, in item 11 in the women control group ( $\sim 6$ seconds, $-7 \%$ p-value 0.039$)$ and in item 14 of the men exercise group ( $\sim 6$ seconds, $-15 \%$ p-value 0.02 ).

No significant differences were observed in the other groups.

\section{Discussion and Directions for Further Research}

Hemiparetic patients of the test group of this study trained only the healthy hand in a short term period of three days with a highly repetitive exercise, such has the NHPT. The paretic-hand-test execution time was lower than before treatment. The reduction was present in each patient confirming the reproducibility of the phenomenon (Tables 6 and 7), meanwhile in the control group only a minimal difference was recorded With this research we can state BT is present in mildly hemiparetic patients and it is not suppressed by stroke, at least in a short time ; the data related to the healthy subjects are similar to the ones collected through patients with stroke: BT phenomenon is more evident in men and more frequent from the dominant hand to the non dominant one, as shown in recent research [19], although it is present in the opposite way with good proficiency [12]. The work of Iosa et al. [19], although the research protocol is very different from ours, being their training time very short, produced results that are similar to ours: i.e. the greater expression of BT phenomenon from the highly skilled hand to the low skilled hand, clearly evident in patients with left hemiparesis. Previous studies in normal subjects indicate that [20-23] BT is likely to be greater if the patient is aware of the general principles that are common to two or more tasks like (A) verbal pre-training, (B) first learning a complex task and then learning a simpler one, (C) spaced, rather than massed, practice, and (D) a high level of motivation, regardless of task interrelationships.
Our results show that men have a greater improvement of the paretic hand performance after BT exercise then women, but the average final speed is similar in both genders (Table 5). In our opinion, there could be an evolutionary explanation, i.e. for more than 4 million years mankind has been organized in societies of hunters and gatherers [24]: perhaps for their hunting and fighting activities men trained for precision mostly the dominant hand, meanwhile women always have been working with both hands for gathering and performing with precision their daily work. Certainly this speculation should be tested on al larger number of normal individuals to give a better significance to this finding.

\begin{tabular}{|l|l|l|l|l|}
\hline Patient & NH i normal & NH f normal & NH i paretic & NH f paretic \\
\hline PT & 57 & 43 & 111 & 67 \\
\hline RL & 47 & 44 & 207 & 202 \\
\hline GG & 40 & 37 & 75 & 47 \\
\hline CR & 54 & 50 & 76 & 62 \\
\hline TR & 55 & 52 & 186 & 153 \\
\hline SD & 33 & 31 & 202 & 160 \\
\hline DE & 38 & 35 & 64 & 44 \\
\hline DA & 33 & 25 & 70 & 32 \\
\hline CG & 55 & 56 & 80 & 62 \\
\hline GG & 36 & 29 & 82 & 69 \\
\hline BC & 50 & 28 & 97 & 77 \\
\hline TF & 36 & 29 & 116 & 86 \\
\hline DC & 51 & 45 & 47 & 47 \\
\hline TL & 50 & 38 & 75 & 54 \\
\hline VG & 57 & 51 & 276 & 178 \\
\hline OV & 42 & 40 & 48 & 41 \\
\hline GR & 45 & 32 & 66 & 61 \\
\hline
\end{tabular}

Table 6: Results of the Test group with exercise: Nine Hole Peg Test

Nine pegs were the inserted in to nine hole of a grid and then removed with the paretic hand, as fast as possible, before and after training only the normal hand.

Nh i normal: Initial record in seconds of the NHPT performed with the normal hand.

Nh $\mathrm{f}$ normal: Final record in seconds of the NHPT performed with the normal hand.

Nh i paretic: Initial record in seconds of the NHPT performed with the paretic hand.

Nh f paretic: Final record in seconds of the NHPT performed with the paretic hand.

BT of skills is shown to be present in stroke patients: it is possible that the repetitive training of a simple task performed by the healthy hand, "teaches" to the paretic hand the motor skill necessary to lower the time of execution of the NHPT. 
Citation: Ausenda CD, Togni G, Biffi M, Morlacchi S, Corrias M, et al. (2014) A New Idea for Stroke Rehabilitation: Bilateral Transfer Analysis from Healthy Hand to the Paretic One with a Randomized and Controlled Trial. Int J Phys Med Rehabil S3: 008. doi: 10.4172/2329-9096.S3-008

Page 7 of 8

These findings can be useful for develop new rehabilitation strategies, in fact they are consistent with the recent growing evidence that the rehabilitation of the upper limb is based on principles of motor learning that include: (I) intense, active repetitive movement; (II) sensorimotor and proprioceptive integration, given the important role that sensory events play on motor learning in the normal and post-stroke states; (III) great importance of attention span and visual control given the effects that these have in normal and neurologically impaired brains [25-29].

All of the results described above and our findings support a model of widespread bi-hemispheric reorganization in the motor system after a focal hemispheric lesion. It is possible to speculate that the brain development in hippocampus region or in sensorial and motors areas observed in these studies, were caused by a greater production of neuronal or glial stem cells that could migrate to the injured cerebral area and could participate to the repair [30]. This growth was noted after improvement in dendritic arborization and synaptic consistency guided by rehabilitation [31].

\begin{tabular}{|l|l|l|l|l|l|l|}
\hline Patient & soll i10 & soll i11 & soll i14 & soll f10 & soll f11 & soll f14 \\
\hline PT & 74 & 22 & 32 & 79 & 24 & 38 \\
\hline RL & 67 & 43 & 33 & 65 & 40 & 32 \\
\hline GG & 48 & 42 & 38 & 37 & 36 & 14 \\
\hline CR & 125 & 48 & 35 & 94 & 42 & 30 \\
\hline TR & 420 & 145 & 320 & 390 & 150 & 142 \\
\hline SD & 100 & 57 & 31 & 90 & 53 & 28 \\
\hline DE & 80 & 48 & 30 & 40 & 42 & 24 \\
\hline DA & 100 & 184 & 22 & 68 & 82 & 21 \\
\hline CG & 90 & 125 & 35 & 93 & 75 & 24 \\
\hline GG & 42 & 38 & 23 & 46 & 37 & 25 \\
\hline BC & 60 & 52 & 31 & 53 & 50 & 29 \\
\hline TF & 104 & 46 & 30 & 99 & 41 & 20 \\
\hline DC & 101 & 28 & 38 & 40 & 20 & 19 \\
\hline TL & 66 & 65 & 42 & 129 & 89 & 32 \\
\hline VG & 95 & 29 & 25 & 82 & 35 & 17 \\
\hline OV & 19 & 29 & 172 & 28 & 27 \\
\hline GR & 19 & 19 & 33 & 27 & 18 \\
\hline
\end{tabular}

Table 7: Results of the Test group: Sollerman's test

Soll i10: Initial record in seconds of Sollerman test Item 10.

Soll i11: Initial record in seconds of Sollerman test Item 11.

Soll i14: Initial record in seconds of Sollerman test Item 14.

Soll f10: Final record in seconds of Sollerman test Item 10.

Soll f11: Final record in seconds of Sollerman test Item 11.

Soll f14: Final record in seconds of Sollerman test Item 14.
The BT described in our study suggests it's not that case, it could raise the hypothesis of an involvement of mirror neurons, because the phenomenon occurs in a short time span.

In monkeys, the mirror neurons [32] the ventral premotor and the rostral inferior parietal neurons are divided in two main categories strictly congruent and broadly congruent. Strictly congruent mirror neurons, one third, fire for the same action, either executed or observed. Broadly congruent mirror neurons representing the remaining two thirds of the mirror neurons fire for actions that are either logically related (such as grasping and bringing to the mouth) or that achieve the same goal, and thus are critical for imitative learning [33].

An interesting FMRI study demonstrated that the human mirror neuron system is critically involved in imitative learning through neural interactions with motor preparation areas and dorsolateral prefrontal cortex [34].

Imitation is a pervasive form of learning in humans, and it is possibly involved in the BT of motor learning that appears from our results, therefore in our opinion, associative cerebral areas, the mirror neurons, are involved in BT.

Instead according to Iosa et al. [19] BT seemed to be related to the development of internal representations, defined as neural functional internal models probably contained in the cerebellum.

The experimental group showed a significant improvement of motor functions compared with both pre-treatment baseline and control groups also in item 10 of the Sollerman test [18], but not in the other items (Table 5).

These results can be used as a "generalization" consequence.

Generalization take places when effects of the therapy spread to other related behaviors and similar movements [26] as recorded in item 10 .

\section{Limitations}

The main limit of this research is the short time span in which the phenomenon has been demonstrated, and another important limit is the small number of participants, in spite of the statistical significance we obtained, the quality of our results would improve with a larger number of patients. But this is not our aim anymore, further research is being performed to define the duration of the phenomenon of bilateral transfer in stroke patients in order to understand if it could be used for developing new rehabilitation strategies. Our experiments are focused on the eventual clinical impact of the BT phenomenon on hand function recovery in stroke survivors.

Another important limitation of this study is the limited number of subjects that could create biased results. In our opinion this problem could affect mainly in the gender difference of the BT phenomenon that is not sufficiently supported by the experiences of previous research on healthy subjects. Meanwhile our results on the side of hemiparesis are consistent with the research of Iosa et al. [19], thus in our opinion they could be considered trustworthy.

It must be underlined that constraint induced motor therapy [34] obtained brilliant results in the rehabilitation of the paretic hand. Moreover a recent review article of van Delden et al. [29] who performed a complete meta-analysis of many recent research protocols suggested that unilateral training is best suited for stroke survivors 
Citation: Ausenda CD, Togni G, Biffi M, Morlacchi S, Corrias M, et al. (2014) A New Idea for Stroke Rehabilitation: Bilateral Transfer Analysis from Healthy Hand to the Paretic One with a Randomized and Controlled Trial. Int J Phys Med Rehabil S3: 008. doi: 10.4172/2329-9096.S3-008

Page 8 of 8

with mild to moderate upper limb hemiparesis. Therefore it is possible that BT will fail to improve the long term recovery of hand function in chronic stroke survivors. As learning and making activities are function of central nervous system, resting a hand and thus limiting fatigue, exercising the other hand could promote new learning at cortical level, shortening recovery times.

\section{Conclusions}

This study shows that BT was present in the patients with the same phenomenon features noted in the healthy subjects, greater incidence among men and a bigger effect when the trained healthy hand is the dominant one, to the paretic non-dominant hand.

BT can offer an alternative or integration to classical rehabilitation methods, helping in preventing fatigue, which affects quantitatively and qualitatively sessions.

\section{Aknowledgment}

Project funded by Regione Lombardia

\section{References}

1. Duncan P, Studenski S, Richards L, Gollub S, Lai SM, et al. (2003) Randomized clinical trial of therapeutic exercise in subacute stroke. Stroke 34: 2173-2180.

2. Hanlon CA, Buffington AL, McKeown MJ (2005) New brain networks are active after right MCA stroke when moving the ipsilesional arm. Neurology 64: 114-120.

3. Woldag H, Lukhaup S, Renner C, Hummelsheim H (2004) Enhanced motor cortex excitability during ipsilateral voluntary hand activation in healthy subjects and stroke patients. Stroke 35: 2556-2559.

4. Dromerick AW, Edwards DF, Hahn M (2000) Does the application of constraint-induced movement therapy during acute rehabilitation reduce arm impairment after ischemic stroke? Stroke 31: 2984-2988.

5. Van Peppen RP, Kwakkel G, Wood-Dauphinee S, Hendriks HJ, Van der Wees PJ, et al. (2004) The impact of physical therapy on functional outcomes after stroke: what's the evidence? Clin Rehabil 18: 833-862.

6. Swift EJ (1903) Studies in the psychology and physiology of learning. Amer J Psychol 14: 201-251.

7. Wieg EL (1932) Bilateral transfer in the motor learning of young children and adults. Child Develop 3: 247-267.

8. Gordon AM, Charles J, Duff SV (1999) Fingertip forces during object manipulation in children with hemiplegic cerebral palsy. II: bilateral coordination. Dev Med Child Neurol 41: 176-185.

9. Ausenda C, Boga F, Mariani E, Restelli E, Sassi L, et al. (2005) Un nuovo metodo riabilitativo per la mano paretica di un paziente affetto da paralisi cerebrale infantile. Casi Clinici in Neuroriabilitazione 6: 10-14.

10. Ausenda C, Carnovali M (2011) Transfer of motor skill learning from the healthy hand to the paretic hand in stroke patients: a randomized controlled trial. Eur J Phys Rehabil Med 47: 417-425.

11. Rinehart JK, Singleton RD, Adair JC, Sadek JR, Haaland KY (2009) Arm use after left or right hemiparesis is influenced by hand preference. Stroke 40: 545-550.

12. Kumar S, Mandal MK (2005) Bilateral transfer of skill in left- and righthanders. Laterality 10: 337-344.
13. Folstein MF, Folstein SE, Mc Hugh PR (1975) "Mini mental state". A practical method for grading the cognitive state of patients for the clinician. J Psychiatr Res 12: 189-198.

14. Mahoney F, Barthel DW (1965) Functional Evaluation: The Barthel Index. Md State Med J 14: 61-65.

15. Bohannon RW, Smith MB (1987) Interrater reliability of a modified Ashworth scale of muscle spasticity. Phys Ther 67: 206-207.

16. Annett M (1970) A classification of hand preference by association analysis. Br J Psychol 61: 303-321.

17. Mathiowetz V, Weber K, Kashman N, Volland G (1985) Adult norms for the Nine hole peg test of finger dexterity. Occup Ther J Res 5: 24-38.

18. Sollerman C, Ejeskär A (1995) Sollerman hand function test. A standardised method and its use in tetraplegic patients. Scand J Plast Reconstr Surg Hand Surg 29: 167-176.

19. Iosa M, Morone G, Ragaglini MR, Fusco A, Paolucci S (2013) Motor strategies and bilateral transfer in sensorimotor learning of patients with subacute stroke and healthy subjects. A randomized controlled trial. Eur J Phys Rehabil Med 49: 291-299.

20. Ammons RB (1958) "Le mouvement" current psychological issues. Henry Holt \& Co, New York.

21. Briggs GE, Brofden WJ (1954) The effect of component practice on performance of a lever-positioning skill. J Exp Psychol 48: 375-380.

22. Cook TW (1933) Studies in cross-education further experiments in mirrors tracing the star-shaped maze. J Exp Psychol 16: 679-700.

23. Allen RM (1948) Factors in mirror drawing. J Educ Psychol 39: 216-226.

24. Bailey GN (1983) Hunter and Gatherer economy in Prehistory: a european perspective. Cambridge University Press, Cambridge UK.

25. Taub E, Miller NE, Novack TA, Cook EW, Fleming WC, et al. (1993) Technique to improve chronic motor deficit after stroke. Arch Phys Med Rehabil 74: 347-354.

26. Takahashi CD, Der-Yeghiaian L, Le V, Motiwala RR, Cramer SC (2008) Robot-based hand motor therapy after stroke. Brain 131: 425-437.

27. Page SJ, Levine P, Leonard A (2007) Mental practice in chronic stroke: results of a randomized, placebo-controlled trial. Stroke 38: 1293-1297.

28. Walker CM, Sunderland A, Sharma J, Walker MF (2004) The impact of cognitive impairment on upper body dressing difficulties after stroke: a video analysis of patterns of recovery. J Neurol Neurosurg Psychiatry 75: 43-48.

29. van Delden AE, Peper CE, Beek PJ, Kwakkel G (2012) Unilateral versus bilateral upper limb exercise therapy after stroke: a systematic review. J Rehabil Med 44: 106-117.

30. Schlegel AA, Rudelson JJ, Tse PU (2012) White matter structure changes as adults learn a second language. J Cogn Neurosci 24: 1664-1670.

31. Gauthier LV, Taub E, Perkins C, Ortmann M, Mark VW, et al. (2008) Remodeling the brain: plastic structural brain changes produced by different motor therapies after stroke. Stroke 39: 1520-1525.

32. Iacoboni M, Mazziotta JC (2007) Mirror neuron system: basic findings and clinical applications. Ann Neurol 62: 213-218.

33. Buccino G, Vogt S, Ritzl A, Fink GR, Zilles K, et al. (2004) Neuron circuits underlying imitation learning of hand actions: an event related FMRI study. Neuron 42: 323-334.

34. Wolf SL, Winstein CJ, Miller JP, Taub E, Uswatte G, et al. (2006) Effect of constraint-induced movement therapy on upper extremity function 3 to 9 months after stroke: the EXCITE randomized clinical trial. JAMA 296: 2095-2104. 